\section{Induced pluripotent stem cells: the long-expected revolution in medical science and practice?}

\author{
Antonio Sorrentino \\ Department of Hematology, Oncology, \\ and Molecular Medicine, Istituto \\ Superiore di Sanità, Rome, Italy
}

\begin{abstract}
Within the matter of a few years, development of the somatic reprogramming technology to generate induced pluripotent stem (iPS) cells has contributed enormously to the stem cell research field. We learned that differentiated adult cells possess an unrestricted plasticity that allows them to be driven back to their embryonic or pluripotent state, but owing to the juvenile nature of this novel science chapter, there are many unanswered questions and dilemmas. It is indisputable, however, that iPS cells potentially could represent the jack-of-alltrades remedy in areas of medicine ranging from toxicology screening to regenerative medicine. In this review I will summarize the current strategies employed to reprogram somatic cells and the major promises and hurdles for the future of iPS cells.
\end{abstract}

\section{Introduction}

An unparalleled achievement in the study of human development and disease modeling was provided by the creation of the first human embryonic stem (hES) cell line in 1998, when Thomson and colleagues derived five hES cell lines from the inner cell mass of blastocysts cultured from donated embryos produced by in vitro fertilization (IVF). ${ }^{1} \mathrm{~A}$ few years later, in August 2006, a major breakthrough in biomedical science was communicated by Takahashi and Yamanaka, describing a straightforward method for generating pluripotent stem cells from embryonic and adult mouse fibroblasts after retroviral transduction of four transcription factors (TFs); namely, Oct-4, Sox-2, Klf-4, and c-Myc (OSKM). ${ }^{2}$ The authors of this seminal work, published in the prestigious Cell journal, named this novel cellular entity induced pluripotent stem (iPS) cells. One year later the same group reported the successful generation of iPS cells from human fibroblasts, ${ }^{3}$ establishing a key landmark in the already boiling-hot area of regenerative medicine: the possibility to travel back in time in patient history and follow disease development from the pluripotent cell.
iPS cells share significant similarities to ES cells in terms of morphology, proliferation, expression of a handful of pluripotency markers, common signaling pathways maintaining the undifferentiated state, and the ability (or hazardous flaw, see below) to form teratocarcinomas in vivo., ${ }^{4,5}$ However, gene expression profiles (mRNAs, microRNAs, and histone modifications) have demonstrated that iPS cells still retain a unique signature, in part owing to differential promoter binding by the reprogramming factors. ${ }^{6}$ Nonetheless, using the OSKM factors, truly pluripotent cells have been generated and when assayed in the most stringent tetraploid complementation assay, that is, injection of foreign diploid ES or iPS cells in extra-embryonic tissue-forming tetraploid blastocysts, they produced viable, reproductively competent donor-derived progeny, ${ }^{7}$ indicating that iPS cells can attain full pluripotency similar to that of ES cells. Stringent criteria and standards have been proposed for the generation of iPS cells to allow cross-laboratory data comparisons, ${ }^{8}$ although some controversy exists on the feasibility of the minimal set of characterization criteria required, especially regarding the true need of in vivo teratoma formation assay (human) and germline competence after chimera formation (mouse) for cells that would be used for in vitro applications only. ${ }^{9}$ Certainly the availability of iPS cells endowed with the ability to derive the three germ layers in vitro but defective of teratoma formation in vivo would be equally (if not superiorly) useful for tissue engineering applications. ${ }^{9}$

\section{Derivation, basic biology, and efficiency of iPS cells reprogramming}

The initial step in the generation of patientspecific iPS cells is to obtain individual tissue samples. iPS cells have been derived from many somatic cell types of origin, typically harvested from tissue biopsy. In addition to the standard dermal fibroblasts, iPS cells have been derived from several sources: mouse hepatocytes and gastric epithelial cells, ${ }^{10}$ human keratinocytes contained in a single plucked hair, which also displayed a greater (>100-fold) and faster (>2-fold) reprogramming efficiency, ${ }^{11}$ cells extracted from exfoliated deciduous teeth, stem cells from the apical papilla, and dental pulp stem cells, ${ }^{12}$ freshly isolated human adipose stem cells in a feederfree condition, ${ }^{13}$ human peripheral blood CD34+ cells, ${ }^{14}$ human cord blood (CB) endothelial-derived cells ${ }^{15}$ and $\mathrm{CD} 133^{+} \mathrm{CB}$ cells, possibly more amenable to reprogramming owing to elevated endogenous expression of Klf-4 and c-
Correspondence: Antonio Sorrentino, Istituto Superiore di Sanità, via Regina Elena 299, Rome 00161, Italy. E-mail: ant_sor@hotmail.com

Key words: embryonic stem cells, induced pluripotency.

Acknowledgements: I thank A. Fatica, M. Valtieri, M. Biffoni, and L.M. Starnes for helpful discussions and critical reading of the manuscript.

Received for publication: 14 January 2010.

Revision received: 3 February 2010.

Accepted for publication: 4 February 2010.

This work is licensed under a Creative Commons Attribution 3.0 License (by-nc 3.0).

CCopyright A. Sorrentino, 2010

Licensee PAGEPress, Italy

Journal of Nucleic Acids Investigation 2010; 1:e1 doi:10.4081/jnai.2010.e1

Myc, ${ }^{16}$ uniparental parthenogenetic neural stem cells, ${ }^{17}$ and adult limbal progenitors isolated from rat limbal epithelium of the cornea. ${ }^{18}$ All of these cell types including skin fibroblasts are potentially clinically relevant, requiring minimal invasive surgical intervention for harvesting. Undoubtedly CB-derived cells could represent one of the most attractive cell types. In particular CB cells are readily accessible and efficient to isolate, they are considered a relatively "young", immunologically immature cell type carrying minimal chromosomal abnormalities and mutations, and probably most importantly, they are bankable, offering a wide selection of HLA haplotypes for transplantation purposes. ${ }^{19}$

The "Yamanaka factors" OSKM" represent the prototypical factors for somatic reprogramming. Equal stoichiometric and temporal expression of the OSKM factors is critical for the successful induction of iPS cells from human fetal fibroblasts. ${ }^{20}$ The individual role of the OSKM TFs has been investigated in the mouse by carrying out a genome-wide analysis of promoter occupancy and expression of Oct4, Sox-2, Klf-4, and c-Myc target genes, ${ }^{21}$ showing that fibroblast markers are repressed and early embryonic markers activated before expression of pluripotency markers. C-Myc is a major contributor to the initial reprogramming event and predominantly acts before pluripotency regulators are activated..$^{21}$ In addition to OSKM, the generation of iPS cells has been achieved by means of various combinations of TFs, arguing against the absolute requirement of some of these factors, particularly c-Myc, for iPS cell formation. In this regard, a screen for candidate reprogramming factors performed in human mesenchymal cells expressing neomycin phosphotransferase driven by the endogenous 0ct-4 promoter to track reprogrammed cells successfully, uncovered that the 
combination 0ct-4/Sox-2/Nanog/Lin28 is sufficient to reprogram foreskin fibroblasts.2 Surprisingly, a factor absolutely required for the establishment of pluripotency, the homeodomain-containing protein Nanog, is not one of the canonical TFs employed to reprogram somatic cells, most likely since its window of action is restrained to the late phases of reprogramming. ${ }^{23}$

Another critical parameter in determining the efficiency and kinetics of reprogramming is the differentiation stage of the cell of origin. ${ }^{24}$ Immature (stem/progenitor) cells have been shown to reprogram faster and more efficiently, possibly because their epigenetic state is more amenable to TF-induced remodeling, ${ }^{24}$ in contrast to previous assumptions ${ }^{25}$ reprogramming does not seem to correlate with proliferation rate. ${ }^{24}$ This notion is supported by the recent finding that adult mouse and human fetal neural stem cells can be reprogrammed by ectopic expression of Oct-4 alone, ${ }^{26,27}$ suggesting that the use of stem/progenitor cells, preferably obtained from CB (see above), might become the elite choice of cell source for reprogramming, since the avoidance of using multiple potential proto-oncogenic TFs would be an important advance in the overall safety of iPS cell generation (see below). A distinct view attests that reprogramming is a continuous stochastic process, where almost all the mouse donor cells eventually give rise to iPS cells on continued growth factor and TFs expression..$^{28}$ Inhibition of the p53/p21 pathway and over-expression of Lin28 increases cell division and accelerates the kinetics of iPS cell formation proportionally to the increase in cell proliferation, while Nanog accelerates reprogramming in a rate-independent cell division. ${ }^{28}$ In fact reprogramming seems to be a stochastic and slow process, suggesting the existence of barriers limiting its efficiency. Senescence is one of these roadblocks: it has been shown that reprogramming triggers up-regulation of $\mathrm{p} 53, \mathrm{p} 16^{\text {inkta }}$, and $\mathrm{p} 21^{\text {cip1 }}$, and therefore senescence, coupled with induction of DNA damage and chromatin remodeling of the INK4a/ARF locus. ${ }^{29}$ Ablation of different senescence effectors improved reprogramming efficiency, although from the perspective of therapeutic application this would be problematic, and transient siRNA-mediated knockdown could be at least a more feasible trial. ${ }^{29}$ These observations have been confirmed in two separate studies demonstrating that iPS cell generation was greatly promoted either in a p53-null background or by pharmacological administration of vitamin $\mathrm{C}$, further proving that the p53-p21 pathway is a critical barrier to iPS cell generation beyond its well-known function in the tumorigenic process. ${ }^{30,31}$

One of the major conceptual and technical challenges in somatic cell reprogramming is the efficiency of iPS cell generation. To date, the standard efficiency of reprogramming from dermal fibroblasts is $\leq 0.01 \%^{3}$ and selecting successfully reprogrammed cells is a tedious process requiring screening of a large number of clones that need to be individually tested for multiple parameters (see above). Several different tools and improvements have been developed to refine this procedure, essentially based on exploiting three basic aspects of iPS/ES cells: epigenetic state, dependence on key signaling pathways, and exogenous TFs delivery requirement (vector design).

Initially, by screening a small collection of known compounds, Shi et al. found that neural progenitor cells could be reprogrammed by using 0ct-4 and Klf-4 TFs in combination with the drug BIX-01294, an inhibitor of the G9a histone methyltransferase, highlighting the importance of appropriate chromatin remodeling during the reprogramming process. ${ }^{32}$ This is also verified by the observation that DNA methyltransferase (DNMT) and histone deacetylase (HDAC) inhibitors improve reprogramming; in particular valproic acid (VA), an HDAC inhibitor, has proven to increase efficiency by more than 100 -fold using 0ct-4, Sox2, and Klf-4 TFs without the introduction of cMyc. ${ }^{33}$ A study employing integrative genomic analysis, including gene expression profiling, chromatin state maps, and DNA methylation analysis of mouse fibroblasts and B lymphocytes undergoing reprogramming, established a conceptual framework in addition to the above observations, showing the existence of a gradient of expression and epigenetic states from the somatic cell to the iPS cell. ${ }^{34}$ Partially reprogrammed cells show DNA hypermethylation at pluripotency-related loci, incomplete repression of lineage-specifying TFs, and reactivation of a distinctive subset of stem-cellrelated genes, while completely reprogrammed iPS cells share similar signatures with ES cells. Overall efficiency could be improved further by inhibition of DNMT1 and incompletely repressed TFs. ${ }^{34}$

The current effort of identifying potential signaling pathways facilitating iPS cell generation relies on the use of chemical screening platforms. A massive high-throughput smallmolecule screening, aimed at identifying compounds $(>500,000)$ that practically could replace Klf-4 in activating Nanog, detected the multikinase inhibitor kenpaullone as a functional substitute for Klf- $4 .{ }^{35}$ A similar approach, although on a much smaller scale, was used to describe an improvement of reprogramming efficiency of $>200$-fold by using the Alk5 inhibitor SB431542, the MEK inhibitor PD0431542, and the pro-survival agent thiazovivin in addition to the canonical OSKM factors. ${ }^{36}$ Inhibition of TGF $\beta$ signaling appears to be a successful strategy, confirmed in two other studies: the TGFBR1 inhibitor E-616452 has been shown to replace Sox-2 in the absence of VA, whereas a second TGFBR1 inhibitor E-616451 and the Src-family kinase inhibitor EI-275 still required VA. ${ }^{37}$ Inhibition of TGF $\beta$ signaling following administration of an Alk5 inhibitor cooperates in the reprogramming of murine fibroblasts, and bypasses the requirement for exogenous c-Myc and Sox-2.38 Finally, an interesting report has shown recently that hypoxia (or in situ normoxia, ${ }^{39}$ i.e. $5 \% \mathrm{O}_{2}$ ) increases the efficiency of iPS cell generation from murine embryonic fibroblasts (MEFs) and bypasses the requirement for Sox2 and $\mathrm{c}-\mathrm{Myc}^{40}$ It is conceivable that the more knowledge of normal stem cell biology is revealed, the more options will be available to find less radical and more "native" implements for somatic reprogramming.

So far, some of the most substantial progress in generating preclinical-grade iPS cells has been obtained in the development of reproducible, efficient, and integration-free methods to introduce the reprogramming factors. Several research groups around the globe are achieving outstanding progress in searching for the optimal combination and delivery of TFs. Initial attempts were made by using nonintegrating adenoviruses transiently expressing the OSKM factors, however reprogramming efficiency seemed lower compared to integrating viruses $;{ }^{41}$ analogous low efficiency was obtained in mouse by using transient transfection with two plasmids expressing Klf4/0ct-4/Sox-2 and c-Myc, respectively. ${ }^{42}$ An interesting attempt, although with the major caveats of very low efficiency, has been made by using a single polycistronic vector encoding Klf-4/0ct-4/Sox-2: the cistron was driven by an EF-1 $\alpha$ promoter in a self-inactivating (SIN) lentiviral vector (LV) containing a LoxP in the 3'LTR, and between each gene was inserted a porcine teschovirus-1 2A sequence triggering ribosome skipping, allowing translation of the multiple coding sequences. Expression of Cre recombinase resulted in deletion of the $\mathrm{LV}$ except for a remnant 291-bp SIN LTR containing a single LoxP site. ${ }^{43}$

A prominent advance in the generation of transgene-free iPS cells has been reached by the use of the piggyBack transposon system: a moth-derived DNA transposon, highly active in mammalian cells and with a very large cargo capacity. ${ }^{44}$ The OSKM factors were linked by sequences encoding $2 \mathrm{~A}$ peptides in a single polycistronic unit, and the resulting transposon vector was introduced in MEFs together with piggyBack transposase expression plasmid to catalyze vector integration. Following generation of primary iPS, the transposon was removed on re-expression of transposase: piggyBack was excised without leaving genetic alterations at the excised site (footprint mutations), and transposon-free iPS cells were selected using the human herpes virus thymidine kinase (HV tk)-fialuridine (FIAU) selec- 
tion system. ${ }^{44}$ This transposon-based delivery method has been perfected further by the creation of a doxycycline-inducible piggyBack plasmid in which, comparably to the previous system, the individual piggyBack insertion can be removed from established iPS cells. ${ }^{45}$ Presumably the instrumental handling of the piggyBack system marks one of the most relevant advances toward achieving clinically suitable methods of generating iPS cells in that it makes use of simple plasmid DNA preparations and delivery, has a broader spectrum of target somatic cell types, grants xeno-free production protocols, and allows accurate transgene removal..$^{45}$ An alternative ingenious method to isolate human iPS cells has been described by Hotta and colleagues using EOS LVs: they engineered a vector containing an early transposon promoter, highly transcribed in ES cells, combined with 0ct-4 and Sox-2 binding motifs in a ES cell-specific core enhancer, plus puromycin resistance gene and EGFP to avoid vector silencing and mark pluripotent stem cells, respectively. ${ }^{46}$ It is worth mentioning another non-integrating episomal expression system derived from the EpsteinBarr virus, the oriP/EBNA1 (Epstein-Barr nuclear antigen-1): this vector can replicate only once per cell cycle, and by using drugselection can be established as a stable episome that could be removed at the end of the process. $^{47}$

Intriguingly, iPS cells have been generated in the absence of viral vectors. In one study, the key step was fusing a poly-arginine (11R) protein transduction domain to the $\mathrm{C}$ terminus of the OSKM factors: these fusion proteins were expressed in $E$. Coli in inclusion bodies that were then solubilized, refolded, and purified. The resulting proteins could be administered directly to the target cells and readily entered the cell membrane after only six hours. $^{48}$ In the second study, the authors exploited the ability of the human immunodeficiency virus transactivator of transcription (HIV-TAT) to cross the cellular membrane. ${ }^{49}$ They initially fused a short segment residing at amino-acids 48-60 of HIV-TAT, containing a high proportion of basic amino-acids (arginine or lysine) and known as CPP, to the OSKM factors. The resulting chimeric fusion protein was expressed in HEK293 and the cell extract was used to treat human newborn fibroblasts. With respect to the previous report, ${ }^{48}$ in this case there was no need to supplement the recombinant proteins with VA for successful reprogramming, and recombinant proteins were produced in a mammalian cell line in contrast to $E$. Coli ${ }^{49}$ The use of soluble proteins instead of viral vectors undoubtedly offers a valid option for deriving iPS cells; on the other hand, the need to repeat the delivery many times and the consequent copious costs of production could represent a serious obstacle to the feasi- bility of the method.

Finally, exogenous administration of ES cellspecific, cell cycle-regulating (ESCC) miRNAs miR-291-3p, miR-294, and miR-295 have been shown to increase the efficiency of reprogramming ${ }^{50}$ offering the attractive opportunity to enhance iPS cell generation by using small RNA-mimicking oligonucleotides.

\section{Regenerative medicine applications: disease modeling and cell-based therapies}

The remarkable yet preliminary therapeutic potential of iPS cells is evident. Albeit ES cells offer the possibility to generate disease-specific pluripotent cells although with severe limitations, at the moment iPS cells offer the prospective of unprecedented disease modeling: to recapitulate the individual patient history faithfully. ${ }^{51}$ iPS cells could be derived from any cell type, fresh or banked, to address the pathogenesis and progression of any genetic disease from the simplest to the more complex. Furthermore, patient-specific iPS cells offer the opportunity for drug screening and discovery and could be used in cell transplantation therapies using HLA-matched or autologous cells. ${ }^{52}$ Besides, it is superfluous to mention that use of iPS cells would avoid the ethically sensible topic of using human embryos or eggs. A further and less explored potential application of iPS cells is their use as a cancer vaccine. A recent study found that human iPS cells can trigger, even if less potently than ES cells, an immune response against a murine colon carcinoma cell line in mice probably through cross-presentation of embryonic antigens. ${ }^{53}$ Both iPS and ES cells were able to immunize naïve mice against challenge with a lethal dose of live colon carcinoma cells and induced a tumor-specific cellular immune response with loss of $\mathrm{CD}_{11 \mathrm{~b}} \mathrm{Gr}^{-1^{+}}$myeloidderived suppressor cells in the spleen. ${ }^{53}$

The record of iPS cell disease-specific models is growing at a constant rate. One of the first proof-of-principle studies for deriving and correcting a disease with autologous iPS cells has been reported for the treatment of sickle cell anemia. ${ }^{54}$ iPS cells were derived from a humanized sickle cell anemia mouse model, the human sickle hemoglobin allele was corrected ex vivo by gene-specific targeting, and transplanted engineered cells partially rescued the disease phenotype. ${ }^{54} \mathrm{~A}$ second interesting study focused on murine hemophilia, a disorder caused by mutations within the Factor VIII (FVIII) gene leading to depleted protein production and inefficient blood clotting. The authors generated iPS cells from tail-tip fibroblasts, differentiated them in vitro in FVIII-pro- ducing endothelial cells and progenitors, and then injected them into the liver of irradiated hemophilia-A mice, showing long-term engraftment and hemophilia phenotype correction. ${ }^{55}$ Ebert et al. generated iPS cells from skin fibroblast samples collected from a child suffering from type I spinal muscular atrophy (SMA).$^{56}$ They showed that the reprogrammed cells retained the disease genotype, consisting mainly of a lack of survival motor neuron 1 (SMA1) gene expression resulting in selective degeneration of lower $\alpha$-motor neurons, and could effectively differentiate in diseased motor neurons and astrocytes..$^{56}$ Disease-specific iPS cells have been derived from reprogrammed dermal fibroblasts obtained from Fanconi anemia (FA) patients. ${ }^{57}$ In this case, restoration of the FA pathway appeared as a prerequisite for iPS cell generation, but genetically corrected cells were indistinguishable from normal iPS cells, in that they were capable of forming phenotypically normal hematopoietic cells. ${ }^{57}$ By using an elegant doxycyclineinducible Cre-recombinase excisable LV system, fibroblasts derived from a patient with idiopathic Parkinson's disease have been reprogrammed and have been shown to differentiate into dopaminergic neurons. ${ }^{58}$ The example offered by Ye et al. is particularly illustrative: they canonically reprogrammed skin fibroblasts from a patient with homozygous $\beta_{0}$-thalassemia into iPS cells, but most importantly they successfully reprogrammed cells from the amniotic fluid or chorionic villus normally used for prenatal diagnosis. ${ }^{59}$ This considerable result opens the opportunity to utilize iPS cells derived after perinatal diagnosis of thalassemia, and use the disease-corrected cells as a treatment in the perinatal periods, instead of the only currently available options of pregnancy termination or logistic support of a child with a life-long illness. ${ }^{59}$

The feasibility of in vitro candidate drug screens using patient-derived iPS cells is especially visible in a study in which iPS cells were generated from fibroblasts of a familial dysautomia patient, a rare but fatal peripheral neuropathy caused by a point mutation in the I-k-B kinase complex-associated protein (IKBKAP) gene.$^{60}$ The above mutation normally results in tissue-specific exon 20 skipping and reduced IKBKAP protein levels causing reduced neuron cells motility, ultimately leading to depletion of autonomic and sensory neurons. Patient-specific iPS cells generated all three germ layers including peripheral neurons containing the tissue-specific mis-splicing of IKBKAP, demonstrating the authentic cellular origin, which recapitulated the disease pathogenesis. ${ }^{60}$ This well-characterized iPS cell model has been used further as an assay for testing candidate drugs affecting IKBKAP levels, showing the feasibility of exploring the disease mechanism and drugs action in 
patient-derived iPS cells. ${ }^{60}$ In the future prospective of cell replacement therapy, iPS cells have been derived from type 1 diabetes patients and have been differentiated into insulin-producing cells. ${ }^{61}$ From this study no evidence emerges on disease recapitulation, in that the derived pancreatic $\beta$-like cells apparently behaved normally, but the problem that pluripotent cells generated from the same patient perform differently surfaces, suggesting incomplete silencing or transgene reactivation and indicating the need of non-viral and more efficient TFs delivery (see below) ${ }^{61}$ Multiple human iPS cell lines have been generated from frozen cord blood or adult CD $34^{+}$ cells from healthy donors or patients with myeloproliferative disorders (MPDs) with acquired JAK2-V617F somatic mutation, which can evolve to polycythemia vera. ${ }^{62}$ Additionally, the reprogrammed cells could be redirected to hematopoietic cells while the MPDs-derived cells recapitulated the disease features in vitro. ${ }^{62}$ Finally, iPS cells from a Duchenne muscular dystrophy (DMD) patient have been derived and corrected by using human artificial chromosome (HAC) carrying the dystrophin gene, providing another valuable proof-of-concept of patient-specific iPS cell's disease modeling. ${ }^{63}$

An additional powerful application of patient-derived iPS cells would be their in vitro differentiation into disease-relevant tissuespecific cells. iPS cells, similar to ES cells, can be maintained stably in an undifferentiated state in vitro and switched to differentiation at any moment. A copious number of welldesigned protocols have been studied to induce tissue-specific differentiation of ES/iPS cells. In addition to the cell types described above, ${ }^{55-57,59-62}$ pluripotent cells have been differentiated into midbrain dopamine and spinal motoneurons without the requirement of embryoid body formation or stromal feeder coculture by targeting SMAD signaling. ${ }^{64}$ Similar to the study by Ebert et al. ${ }^{56}$ neural differentiation has been obtained from iPS cells derived from a patient with amyotrophic lateral sclerosis (ALS). ${ }^{65}$ Another two interesting studies respectively described specification of retinal cells containing functional photoreceptors ${ }^{66}$ and hepatic endoderm-derived hepatocytes. ${ }^{67}$

It is tempting to speculate that iPS cellderived differentiated cells one day could regenerate damaged tissues and organs either by implanting engineered in vitro-produced tissues into the patient or by directly replacing injured tissues in their native environment in vivo. This could even lead to whole organ replacement in the contingency of end-organ failure. Most likely the creation of transplantable tissues will require the optimization of bioactive scaffolds providing the appropriate biomechanical microenvironment to generate sufficient clinical-scale quantities of cells. ${ }^{68}$
Genetic modification of patient-derived iPS cells and derivatives, including disease correction or tracking, would be the ultimate goal of the theoretical disease modeling workflow. Various viral vectors have been exploited as tools to achieve exogenous expression of transgenes in iPS or ES cells, although with limited efficiency or selectivity. ${ }^{69}$ Thankfully, advances in gene targeting of endogenous loci are rapidly developing. The host-factor independent transposon piggyBack has been engineered to create fully reversible genetic modifications in ES cells to generate clinical-grade ES cell derivatives. ${ }^{70}$ This newly created ePiggyBack can deliver up to $18 \mathrm{~kb}$ of genetic material, transgene expression is highly efficient and, most importantly, can be readily remobilized to a recipient plasmid by reexpression of transposase. Compared to Creor Flp- and Sleeping Beauty-based systems, ePiggyBack removal does not leave any mutations in the host genome, even though transgene integration patterns are still random. ${ }^{70}$

Originally developed by Naldini's group, the "genome editing" technique, based on integrase-defective LVs (IDLVs) expressing zincfinger nucleases (ZFNs) to target specific loci in stem cells by homologous recombination (HR),${ }^{71}$ is evolving as a promising tool to target genes in ES and iPS cells. Well known to researchers in the field, human iPS cells grow poorly as single cells, a practice required to select rare targeted clones using plasmidbased HR. A recent work described a virus-free system to perform ZFN-mediated gene targeting at the endogenous PIG-A locus, a gene normally mutated in hematopoietic stem cells from patients with paroxysmal nocturnal hemoglobinuria (PNH). ${ }^{72}$ By using ZFNs, HRmediated gene targeting ensured permanent genetic alterations, increased rate of targeting efficiency, high specificity, and no karyotypic abnormalities. ${ }^{72}$ A similar technique was perfected to create an efficient method for genetic modification using ZFN-mediated genome targeting, achieving superior site-specific integration of exogenous genes under control of either constitutively active, inducible, or tissue-specific promoters. ${ }^{73}$

\section{Safety concerns}

Since Takahashi and Yamanaka reported the generation of iPS cells for the first time, for many scientists somatic reprogramming was and still is surrounded by a nimbus of skepticism, for a good reason: the OSKM factors are proto-oncogenes. This is especially noticeable for c-Myc, a well-known oncogene deregulated in $70 \%$ of all cancers. ${ }^{74}$ Several findings argue that c-Myc is not absolutely required for iPS cell generation, although the absence of the TF substantially reduces efficiency and results in longer latency of reprogramming. ${ }^{75}$ By crossing adult chimeras, obtained from iPS cell clones derived using the OSKM factors and selected for Nanog expression, germline transmission is achievable; however, approximately $20 \%$ of the offspring developed ganglioneuroblastoma with follicular carcinomas caused by c-Myc reactivation. ${ }^{76}$ Later, the same authors found that the use of c-Myc does not affect the teratoma-forming propensity of secondary neurospheres (SNS) derived from iPS cells, and no c-Myc or other transgene reactivation was observed in SNS or teratomas. ${ }^{77}$ The authors reasoned that teratoma formation propensity varies significantly depending on the iPS cell's tissue of origin, showing the greater susceptibility when iPS cells are derived from adult tail-tip fibroblasts, followed by hepatocytes, MEFs, and gastric epithelial cells. ${ }^{77}$ Unfortunately, even excluding the use of c-Myc we are far from feeling safe and sound: induced expression of Oct- 4 or Klf- 4 in the adult mouse epithelial compartment results in dysplasia, ${ }^{78,79}$ while Sox-2 expression is increased in serrated polyps and mucinous colon carcinomas. ${ }^{80}$ The sole use of integrating viral vector systems may result in insertional mutagenesis evolving in malignant transformation. $^{81}$

The two-sided nature of the OSKM factors, stem cell and oncogenic factors, inevitably highlights the principle that pluripotency and tumorigenicity are bound together, and that iPS cell induction shares similar features to oncogenic progression..$^{82}$ Indeed, acquisition of immortality is a crucial and rate-limiting step in the establishment of the pluripotent state in somatic cells, in particular the epigenetic silencing of the INK4a/ARF locus has been shown to be a critical step for the conversion to iPS cells, and is a major contributor to the low efficiency and delayed kinetics of in vitro reprogramming ${ }^{83}$

Improvements in the protocols for generation and characterization of iPS cells will increase safety until researchers are able to find a way to separate "stemness" from tumorigenicity. Genetically homogenous mice carrying different combinations of doxycycline-inducible reprogramming factors at minimal copy number have been generated, representing a highly valuable tool to study the reprogramming mechanism and to perform high-throughput drug screens. ${ }^{84}$ Such transgenic systems would also cut down genetic variability caused by random integration of multiple proviral copies during iPS cell generation using integrating-vectors. ${ }^{84}$ Xeno-free iPS cells from human dermal fibroblasts have been generated very recently, eliminating batch-to-batch variability of human serum, ${ }^{85}$ and together with the finding that iPS cells can be produced on isogenic parental fibroblasts as 
feeders ${ }^{86}$ is contributing to the stepwise establishment of clinical-grade cells. Using live immunofluorescence staining and flow cytometry time course analysis of fibroblasts undergoing reprogramming, Chan and colleagues identified fully reprogrammed cells over reprogramming intermediates based on TRA-1-60, DNMT38, Rex1 expression, and proviral silencing, thus establishing more rigorous standardization and characterization of iPS cells. ${ }^{87}$

\section{Conclusions}

At the beginning of 2009, almost simultaneously to President Obama's inaugural speech, the U.S. Food and Drug Administration (FDA) cleared the first Phase I clinical trial involving the use of hES cells to target acute spinal cord injury (www.geron.com). Shortly afterward, the Maryland biotech NeuralStem obtained the approval by the FDA for a clinical trial to test hES cells in patients with Lou Gehrig's disease (www.neuralstem.com), whereas Advanced Cell Technology will soon initiate a Phase I/II multicenter trial using hES cells to treat patients with Stargardt's Macular Dystrophy (SMD) (www.advancedcell.com). It is conceivable that in the next few years (perhaps decades) scientists will achieve conclusive progress in understanding the complexity of somatic reprogramming, so that iPS cells will substitute ES cells in most clinical settings. At present this is still considerably faroff, yet staggeringly promising.

\section{References}

1. Thomson JA, Itskovitz-Eldor J, Shapiro SS, et al. Embryonic stem cell lines derived from human blastocysts. Science 1998; 282:1145-7.

2. Takahashi K, Yamanaka S. Induction of pluripotent stem cells from mouse embryonic and adult fibroblast cultures by defined factors. Cell 2006;126:663-76.

3. Takahashi K, Tanabe K, Ohnuki M, et al. Induction of pluripotent stem cells from adult human fibroblasts by defined factors. Cell 2007;131:861-72.

4. Vallier L, Touboul T, Brown S, et al. Signaling pathways controlling pluripotency and early cell fate decisions of human induced pluripotent stem cells. Stem Cells 2009;27:2655-66.

5. Colman A, Dreesen 0. Induced pluripotent stem cells and the stability of the differentiated state. EMBO Reports 2009;10:71421.

6. Chin MH, Mason MJ, Xie W, et al. Induced pluripotent stem cells and embryonic stem cells are distinguished by gene expression signatures. Cell Stem Cell 2009;5:111-23.

7. Zhao XY, Li W, Lv Z, et al. iPS cells produce viable mice through tetraploid complementation. Nature 2009;461:86-90.

8. Maherali N, Hochedlinger K. Guidelines and techniques for the generation of induced pluripotent stem cells. Cell Stem Cell 2008;3:595-605.

9. Ellis J, Bruneau BG, Keller G, et al. Alternative induced pluripotent stem cell characterization criteria for in vitro applications. Cell Stem Cell 2009;4:198-9; author reply 202.

10. Aoi T, Yae K, Nakagawa $M$, et al. Generation of pluripotent stem cells from adult mouse liver and stomach cells. Science 2008;321:699-702.

11. Aasen T, Raya A, Barrero MJ, et al. Efficient and rapid generation of induced pluripotent stem cells from human keratinocytes. Nat Biotechnol 2008;26:127684.

12. Yan X, Qin H, Qu C, et al. iPS cells reprogrammed from mesenchymal-like stem/ progenitor cells of dental tissue origin. Stem Cells Dev 2009 0ct 1.

13. Sun N, Panetta NJ, Gupta DM, et al. Feeder-free derivation of induced pluripotent stem cells from adult human adipose stem cells. Proc Nat Acad Sci USA 2009; 106:15720-5.

14. Loh YH, Agarwal S, Park IH, et al. Generation of induced pluripotent stem cells from human blood. Blood 2009;113:5476-9.

15. Haase A, Olmer R, Schwanke K, et al. Generation of induced pluripotent stem cells from human cord blood. Cell Stem Cell 2009;5:434-41.

16. Giorgetti A, Montserrat N, Aasen T, et al. Generation of induced pluripotent stem cells from human cord blood using 0CT4 and SOX2. Cell Stem Cell 2009;5:353-7.

17. Do JT, Joo JY, Han DW, et al. Generation of parthenogenetic iPS cells from parthenogenetic neural Stem Cells. Stem Cells 2009:27:2962-8.

18. Balasubramanian S, Babai N, Chaudhuri A, et al. Non cell-autonomous reprogramming of adult ocular progenitors: generation of pluripotent stem cells without exogenous transcription factors. Stem Cells 2009; 27:3053-62.

19. Ruhil S, Kumar V, Rathee P. Umbilical cord stem cell: an overview. Curr Pharmaceut Biotechnol 2009;10:327-34.

20. Papapetrou EP, Tomishima MJ, Chambers SM, et al. Stoichiometric and temporal requirements of Oct4, Sox2, Klf4, and cMyc expression for efficient human iPSC induction and differentiation. Proc Nat Acad Sci USA 2009;106:12759-64.

21. Sridharan R, Tchieu J, Mason MJ, et al.
Role of the murine reprogramming factors in the induction of pluripotency. Cell 2009;136:364-77.

22. Yu J, Vodyanik MA, Smuga-Otto K, et al. Induced pluripotent stem cell lines derived from human somatic cells. Science 2007;318:1917-20.

23. Silva J, Nichols J, Theunissen TW, et al. Nanog is the gateway to the pluripotent ground state. Cell 2009;138:722-37.

24. Eminli S, Foudi A, Stadtfeld M, et al. Differentiation stage determines potential of hematopoietic cells for reprogramming into induced pluripotent stem cells. Nat Genet 2009;41:968-76.

25. Jaenisch R, Young R. Stem cells, the molecular circuitry of pluripotency and nuclear reprogramming. Cell 2008;132: 567-82.

26. Kim JB, Sebastiano V, Wu G, et al. Oct4induced pluripotency in adult neural stem cells. Cell 2009;136:411-9.

27. Kim JB, Greber B, Arauzo-Bravo MJ, et al. Direct reprogramming of human neural stem cells by OCT4. Nature 2009;461:6493.

28. Hanna J, Saha K, Pando B, et al. Direct cell reprogramming is a stochastic process amenable to acceleration. Nature 2009; 462:595-601.

29. Banito A, Rashid ST, Acosta JC, et al. Senescence impairs successful reprogramming to pluripotent stem cells. Genes Dev 2009;23:2134-9.

30. Hong H, Takahashi K, Ichisaka T, et al. Suppression of induced pluripotent stem cell generation by the p53-p21 pathway. Nature 2009;460:1132-5.

31. Esteban MA, Wang T, Qin B, et al. Vitamin $\mathrm{C}$ enhances the generation of mouse and human induced pluripotent stem cells. Cell Stem Cell 2010;6:71-9.

32. Shi Y, Do JT, Desponts C, et al. A combined chemical and genetic approach for the generation of induced pluripotent stem cells. Cell Stem Cell 2008;2:525-8.

33. Huangfu D, Maehr R, Guo W, et al. Induction of pluripotent stem cells by defined factors is greatly improved by small-molecule compounds. Nat Biotechnol 2008;26:795-7.

34. Mikkelsen TS, Hanna J, Zhang X, et al. Dissecting direct reprogramming through integrative genomic analysis. Nature 2008;454:49-55.

35. Lyssiotis CA, Foreman RK, Staerk J, et al. Reprogramming of murine fibroblasts to induced pluripotent stem cells with chemical complementation of Klf4. Proc Nat Acad Sci USA 2009;106:8912-7.

36. Lin T, Ambasudhan R, Yuan X, et al. A chemical platform for improved induction of human iPSCs. Nat Methods 2009;6:8058 . 
37. Ichida JK, Blanchard J, Lam K, et al. A small-molecule inhibitor of tgf-Beta signaling replaces sox2 in reprogramming by inducing nanog. Cell Stem Cell 2009;5:491503.

38. Maherali N, Hochedlinger K. Tgfbeta signal inhibition cooperates in the induction of iPSCs and replaces Sox2 and cMyc. Curr Biol 2009;19:1718-23.

39. Ivanovic Z. Hypoxia or in situ normoxia: The stem cell paradigm. J Cell Physiol 2009;219:271-5.

40. Yoshida Y, Takahashi K, Okita K, et al. Hypoxia enhances the generation of induced pluripotent stem cells. Cell Stem Cell 2009;5:237-41.

41. Stadtfeld M, Nagaya M, Utikal J, et al. Induced pluripotent stem cells generated without viral integration. Science 2008; 322:945-9.

42. Okita K, Nakagawa M, Hyenjong $\mathrm{H}$, et al. Generation of mouse induced pluripotent stem cells without viral vectors. Science 2008;322:949-53.

43. Chang CW, Lai YS, Pawlik KM, et al. Polycistronic lentiviral vector for "hit and run" reprogramming of adult skin fibroblasts to induced pluripotent stem cells. Stem Cells 2009;27:1042-9.

44. Yusa K, Rad R, Takeda J, et al. Generation of transgene-free induced pluripotent mouse stem cells by the piggyBac transposon. Nat Methods 2009;6:363-9.

45. Woltjen K, Michael IP, Mohseni P, et al. piggyBac transposition reprograms fibroblasts to induced pluripotent stem cells. Nature 2009;458:766-70.

46. Hotta A, Cheung AY, Farra N, et al. Isolation of human iPS cells using EOS lentiviral vectors to select for pluripotency. Nat Methods 2009;6:370-6.

47. Yu J, Hu K, Smuga-Otto K, et al. Human induced pluripotent stem cells free of vector and transgene sequences. Science 2009;324:797-801.

48. Zhou H, Wu S, Joo JY, et al. Generation of induced pluripotent stem cells using recombinant proteins. Cell Stem Cell 2009; 4:381-4.

49. Kim D, Kim CH, Moon JI, et al. Generation of human induced pluripotent stem cells by direct delivery of reprogramming proteins. Cell Stem Cell 2009;4:472-6.

50. Judson RL, Babiarz JE, Venere M, et al. Embryonic stem cell-specific microRNAs promote induced pluripotency. Nat Biotechnol 2009;27:459-61.

51. Colman A, Dreesen 0. Pluripotent stem cells and disease modeling. Cell Stem Cell 2009;5:244-7.

52. Duinsbergen D, Salvatori D, Eriksson M, et al. Tumors originating from induced pluripotent stem cells and methods for their prevention. Ann NY Acad Sci 2009;
1176:197-204.

53. Li Y, Zeng H, Xu RH, et al. Vaccination with human pluripotent stem cells generates a broad spectrum of immunological and clinical response against colon cancer. Stem Cells 2009;27:3103-11.

54. Hanna J, Wernig M, Markoulaki S, et al. Treatment of sickle cell anemia mouse model with iPS cells generated from autologous skin. Science 2007;318:1920-3.

55. Xu D, Alipio Z, Fink LM, et al. Phenotypic correction of murine hemophilia A using an iPS cell-based therapy. Proc Nat Acad Sci USA 2009;106:808-13.

56. Ebert AD, Yu J, Rose FF Jr, et al. Induced pluripotent stem cells from a spinal muscular atrophy patient. Nature 2009;457: 277-80.

57. Raya A, Rodriguez-Piza I, Guenechea G, et al. Disease-corrected haematopoietic progenitors from Fanconi anaemia induced pluripotent stem cells. Nature 2009;460: 53-9.

58. Soldner F, Hockemeyer D, Beard C, et al. Parkinson's disease patient-derived induced pluripotent stem cells free of viral reprogramming factors. Cell 2009;136:96477.

59. Ye L, Chang JC, Lin C, et al. Induced pluripotent stem cells offer new approach to therapy in thalassemia and sickle cell anemia and option in prenatal diagnosis in genetic diseases. Proc Nat Acad Sci USA 2009;106:9826-30.

60. Lee G, Papapetrou EP, Kim H, et al. Modelling pathogenesis and treatment of familial dysautonomia using patient-specific iPSCs. Nature 2009;461:402-6.

61. Maehr R, Chen S, Snitow M, et al. Generation of pluripotent stem cells from patients with type 1 diabetes. Proc Nat Acad Sci USA 2009;106:15768-73.

62. Ye Z, Zhan H, Mali P, et al. Human induced pluripotent stem cells from blood cells of healthy donors and patients with acquired blood disorders. Blood 2009;114:5473-80.

63. Kazuki Y, Hiratsuka M, Takiguchi M, et al. Complete genetic correction of iPS cells from duchenne muscular dystrophy. Mol Ther 2010;18:238-40.

64. Chambers SM, Fasano CA, Papapetrou EP, et al. Highly efficient neural conversion of human ES and iPS cells by dual inhibition of SMAD signaling. Nat Biotechnol 2009;27:275-80.

65. Dimos JT, Rodolfa KT, Niakan KK, et al. Induced pluripotent stem cells generated from patients with ALS can be differentiated into motor neurons. Science 2008;321: 1218-21.

66. Osakada F, Jin ZB, Hirami Y, et al. In vitro differentiation of retinal cells from human pluripotent stem cells by small-molecule induction. J Cell Sci 2009;122:3169-79.
67. Sullivan GJ, Hay DC, Park IH, et al. Generation of functional human hepatic endoderm from human induced pluripotent stem cells. Hepatology 2010;51:32935 .

68. Polak JM, Bishop AE. Stem cells and tissue engineering: past, present, and future. Ann NY Acad Sci 2006;1068:352-66.

69. Giudice A, Trounson A. Genetic modification of human embryonic stem cells for derivation of target cells. Cell Stem Cell 2008;2:422-33.

70. Lacoste A, Berenshteyn F, Brivanlou AH. An efficient and reversible transposable system for gene delivery and lineage-specific differentiation in human embryonic stem cells. Cell Stem Cell 2009;5:332-42.

71. Lombardo A, Genovese P, Beausejour CM, et al. Gene editing in human stem cells using zinc finger nucleases and integrasedefective lentiviral vector delivery. Nat Biotechnol 2007;25:1298-306.

72. Zou J, Maeder ML, Mali P, et al. Gene targeting of a disease-related gene in human induced pluripotent stem and embryonic stem cells. Cell Stem Cell 2009;5:97-110.

73. Hockemeyer D, Soldner F, Beard C, et al. Efficient targeting of expressed and silent genes in human ESCs and iPSCs using zinc-finger nucleases. Nat Biotechnol 2009;27:851-7.

74. Meyer N, Penn LZ. Reflecting on 25 years with MYC. Nat Rev Cancer 2008;8:976-90.

75. Yamanaka S. A fresh look at iPS cells. Cell 2009;137:13-7.

76. Okita K, Ichisaka T, Yamanaka S. Generation of germline-competent induced pluripotent stem cells. Nature 2007;448: 313-7.

77. Miura K, Okada Y, Aoi T, et al. Variation in the safety of induced pluripotent stem cell lines. Nat Biotechnol 2009;27:743-5.

78. Hochedlinger K, Yamada Y, Beard C, et al. Ectopic expression of Oct-4 blocks progenitor-cell differentiation and causes dysplasia in epithelial tissues. Cell 2005;121:46577.

79. Foster KW, Liu Z, Nail CD, et al. Induction of KLF4 in basal keratinocytes blocks the proliferation-differentiation switch and initiates squamous epithelial dysplasia. Oncogene 2005;24:1491-500.

80. Park ET, Gum JR, Kakar S, et al. Aberrant expression of S0X2 upregulates MUC5AC gastric foveolar mucin in mucinous cancers of the colorectum and related lesions. Int J Cancer 2008;122:1253-60.

81. Romano G, Marino IR, Pentimalli F, et al. Insertional mutagenesis and development of malignancies induced by integrating gene delivery systems: implications for the design of safer gene-based interventions in patients. Drug News Perspect 2009;22: 185-96. 
82. Knoepfler PS. Deconstructing stem cell tumorigenicity: a roadmap to safe regenerative medicine. Stem Cells 2009;27:10506.

83. Utikal J, Polo JM, Stadtfeld M, et al. Immortalization eliminates a roadblock during cellular reprogramming into iPS cells. Nature 2009;460:1145-8.

84. Markoulaki S, Hanna J, Beard C, et al.
Transgenic mice with defined combinations of drug-inducible reprogramming factors. Nat Biotechnol 2009;27:169-71.

85. Rodriguez-Piza I, Richaud-Patin Y, Vassena R, et al. Reprogramming of human fibroblasts to induced pluripotent stem cells under xeno-free Conditions. Stem Cells 2010;28:36-44.

86. Takahashi K, Narita M, Yokura M, et al.
Human induced pluripotent stem cells on autologous feeders. PloS 2009;4:e8067.

87. Chan EM, Ratanasirintrawoot S, Park IH, et al. Live cell imaging distinguishes bona fide human iPS cells from partially reprogrammed cells. Nat Biotechnol 2009;27: 1033-7. 